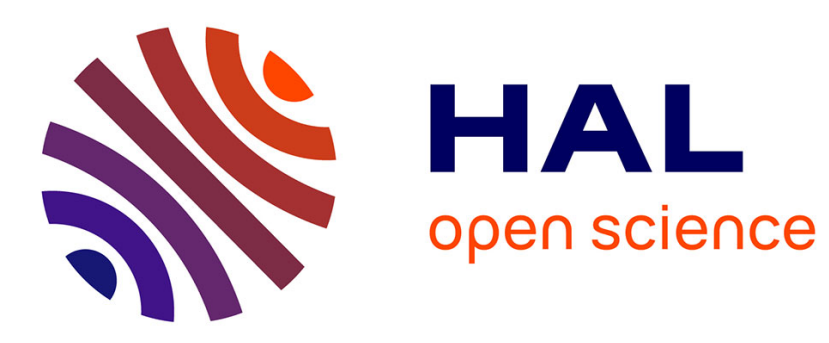

\title{
Fluid description of neutral particles in divertor regimes in WEST
}

Matteo Valentinuzzi, Giorgio Giorgiani, Yannick Marandet, Hugo Bufferand, Guido Ciraolo, Ph. Ghendrih, Patrick Tamain, Eric Serre, Philippe Ghendrih

\section{- To cite this version:}

Matteo Valentinuzzi, Giorgio Giorgiani, Yannick Marandet, Hugo Bufferand, Guido Ciraolo, et al.. Fluid description of neutral particles in divertor regimes in WEST. Contributions to Plasma Physics, 2018, 58 (6-8), pp.710-717. 10.1002/ctpp.201700211 . hal-02116176

\section{HAL Id: hal-02116176 \\ https://hal-amu.archives-ouvertes.fr/hal-02116176}

Submitted on 30 Apr 2019

HAL is a multi-disciplinary open access archive for the deposit and dissemination of scientific research documents, whether they are published or not. The documents may come from teaching and research institutions in France or abroad, or from public or private research centers.
L'archive ouverte pluridisciplinaire HAL, est destinée au dépôt et à la diffusion de documents scientifiques de niveau recherche, publiés ou non, émanant des établissements d'enseignement et de recherche français ou étrangers, des laboratoires publics ou privés. 


\title{
Fluid description of neutral particles in divertor regimes in WEST
}

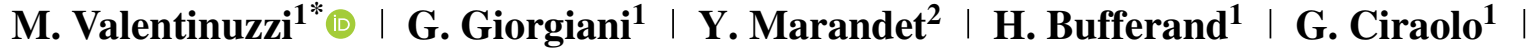 \\ P. Tamain ${ }^{1}$ | E. Serre ${ }^{3}$ | Ph. Ghendrih ${ }^{1}$
}

${ }^{1}$ CEA, IRFM, Saint-Paul-Lez-Durance, France ${ }^{2}$ CNRS, Laboratoire PIIM, Aix Marseille University, Marseille, France

${ }^{3}$ CNRS, Ecole Centrale, Laboratoire M2P2, Aix Marseille University, Marseille, France

*Correspondence

M. Valentinuzzi, CEA, IRFM, F-13108

Saint-Paul-Lez-Durance, France.

Email: matteo.valentinuzzi@cea.fr

Funding Information

This research was supported by the Agence

Nationale de la Recherche, ANR-10-EQPX-29-01. H2020 Euratom, No. 633053.
A new neutral fluid code has been developed as a necessary step towards a hybrid fluid/kinetic neutral model, to be used in ITER or DEMO simulations, where part of the divertor will be very collisional for neutrals. The neutral fluid code, which is able to handle complex geometries in view of the coupling to Soledge2D, is tested on plasma backgrounds obtained by Soledge2D-Eirene in WEST geometry, for different divertor regimes, and is found to be in fair agreement with the kinetic Monte Carlo solver Eirene. The differences are due to the simplifications introduced in the fluid model and to the fact that a fluid description is not fully valid in these cases.

KEYWORDS

computational fluid dynamics, hybrid model, transport

\section{1 | INTRODUCTION}

Power exhaust is one of the major challenges of future devices such as ITER and DEMO. Because of the lack of identified scaling parameters, predictions for divertor plasma conditions in these devices have to rely on edge transport codes, which often consist of a fluid code for the plasma (such as Soledge2 $\mathrm{D}^{[1]}$ ) coupled to a kinetic Monte Carlo code (such as Eirene ${ }^{[2]}$ ) for the neutral particles. The latter incorporates the complex atomic, molecular, and surface processes characteristic of edge plasmas. The use of a kinetic description for the neutral gas stems from the fact that in most of the devices the ratio of the neutrals' mean free path to a representative physical length scale (the Knudsen number, $K n$ ) is much larger than 1 . However, in the divertor region close to the neutralizer target plates, especially for large machines as ITER or DEMO, the situation can be very different owing to high density of the order of $10^{20}-10^{21} \mathrm{~m}^{-3}$ and low temperatures, below $5 \mathrm{eV}$. In this regime, the kinetic description is too detailed (locally $K n \ll 1$ ) and the codes tend to be very inefficient because of the Monte Carlo approach.

A hybrid model then becomes appealing for the neutral gas, treating the latter as a collisional fluid in low-temperature and high-density areas and kinetically elsewhere. In order to achieve this, a new fluid neutrals code has been developed, which is described in Section 2; the results of three simulations in WEST geometry carried out with Soledge2D-Eirene are shown in Section 3, and comparisons are drawn with similar simulations performed with the fluid code in Section 4; in Section 5, the impact of the lack of molecules in the fluid code is assessed, and, finally, conclusions and perspectives are discussed in Section 6.

\section{2 | FLUID NEUTRALS CODE}

The fluid code for neutrals solves the system of equations

$$
\begin{aligned}
& \frac{\partial n_{n}}{\partial t}+\vec{\nabla} \cdot\left(n_{n} \vec{u}_{n}\right)=S_{n} \\
& \frac{\partial n_{n} \vec{u}_{n}}{\partial t}+\vec{\nabla} \cdot\left(n_{n} \vec{u}_{n} \otimes \vec{u}_{n}+\frac{p_{n}}{m_{n}} I\right)=\vec{S}_{\mathrm{mom}},
\end{aligned}
$$


TABLE 1 Atomic and molecular reactions used in Eirene (kinetic code, on the left) and in the fluid code (on the right)

\begin{tabular}{lcc} 
Reaction & Eirene & Fluid code \\
\hline $\mathrm{D}^{0}+\mathrm{e}^{-} \Rightarrow 2 \mathrm{e}^{-}+\mathrm{D}^{+}$ & $\sqrt{ }$ & $\sqrt{ }$ \\
$\mathrm{D}^{+}+\mathrm{e}^{-} \Rightarrow \mathrm{D}^{0}$ & $\sqrt{ }$ & $\sqrt{ }$ \\
$\mathrm{D}^{0}+\mathrm{D}^{+} \Rightarrow \mathrm{D}^{+}+\mathrm{D}^{0}$ & $\sqrt{ }$ & $\sqrt{ }$ \\
$\mathrm{D}_{2}+\mathrm{e}^{-} \Rightarrow 2 \mathrm{D}^{0}+\mathrm{e}^{-}$ & $\sqrt{ }$ & - \\
$\mathrm{D}_{2}+\mathrm{e}^{-} \Rightarrow \mathrm{D}_{2}^{+}+2 \mathrm{e}^{-}$ & $\sqrt{ }$ & - \\
$\mathrm{D}_{2}+\mathrm{e}^{-} \Rightarrow \mathrm{D}^{0}+\mathrm{D}^{+}+2 \mathrm{e}^{-}$ & $\sqrt{ }$ & - \\
$\mathrm{D}_{2}^{+}+\mathrm{e}^{-} \Rightarrow \mathrm{D}^{0}+\mathrm{D}^{0 *}$ & $\sqrt{ }$ & - \\
$\mathrm{D}_{2}^{+}+\mathrm{e}^{-} \Rightarrow 2 \mathrm{D}^{+}+2 \mathrm{e}^{-}$ & $\sqrt{ }$ & - \\
$\mathrm{D}_{2}^{+}+\mathrm{e}^{-} \Rightarrow \mathrm{D}^{0}+\mathrm{D}^{+}+\mathrm{e}^{-}$ & $\sqrt{ }$ & - \\
$\mathrm{D}_{2}^{+}+\mathrm{e}^{-} \Rightarrow 2 \mathrm{D}^{0}$ & $\sqrt{ }$ & - \\
\hline
\end{tabular}

where $n_{n}=\int f d \vec{v}$ is the neutral density, $\vec{u}_{n}=\left(u_{R}, u_{Z}, u_{\phi}\right)=\frac{1}{n_{n}} \int f \vec{v} d \vec{v}$ is the neutral fluid velocity, and $p_{n}=n_{n} T_{n}=n_{n} T_{i}$ is the neutral pressure. The underlying assumption for the closure of the model is that the neutrals are described by a Maxwellian distribution function with temperature $T_{n}=T_{i}$. The particle $S_{n}$ and momentum $\vec{S}_{\text {mom }}$ source terms are due to the interactions between neutrals and the plasma.

Only hydrogen $(\mathrm{H}, \mathrm{D}$, or T) atoms are taken into account in the current version of the code, with only a reduced set of reactions implemented as shown in Table 1 because of the validity of the fluid description in, e.g. charge-exchange-dominated regimes. Additionally, since the focus of this paper is not on the molecules, Eirene simulations in the following include only a crude treatment of molecular reactions, especially for detachment studies, with respect to more detailed models. ${ }^{[3]}$ Furthermore, the assumption of toroidal symmetry $\frac{\partial\langle\cdot\rangle}{\partial \phi}=0$ is used to reduce the problem to two spatial dimensions ( $R$ and $Z$ ).

The boundaries of the computational grid are defined on the core-edge interface and at the wall. At the core surface, it is assumed that all neutrals that cross this surface get ionized and are effectively absorbed. At the wall, instead, a fraction $R_{n}$ of the neutral flux impacting on the solid surface is reflected, and a fraction $R_{i}$ of the ion flux is recycled; only the "fast" part of the recycling fraction, because of instantaneous back-scattering, is taken into account, while the absorbed and later thermally-desorbed-as-molecules part is not implemented. For simplicity, the effective flux $\Gamma_{n}=R_{i} \Gamma_{i}^{-}+\left(R_{n}-1\right) \Gamma_{n}^{-}$is imposed as going out of the wall along the normal vector (taken as pointing towards the core, and the superscripts + and - for the fluxes refer to this direction) and with zero toroidal component.

From the numerical point of view, the code has been implemented with a hybridizable discontinuous Galerkin (HDG) method, ${ }^{[4,5]}$ which has various advantages such as reduction of the number of degrees of freedom of the numerical problem, the possibility of using arbitrary meshes (in particular, the same mesh used by Eirene), and stable high-order discretization of convective and diffusive operators. Finally, the coupling with Soledge2D-Eirene allows the code to simulate the neutrals in complex geometries, as the realistic wall and volume description is crucial for the determination of the neutral pressure.

\section{3 | WEST DIVERTOR REGIMES WITH SOLEDGE2D-EIRENE}

Three different simulations were run with Soledge2D-Eirene in WEST geometry to assess the role of neutral particles in three different divertor regimes. These simulations were run with similar input parameters and boundary conditions: pure deuterium plasmas; lower single null configuration in the so-called far scenario; ${ }^{[6]} 4 \mathrm{MW}$ of power coming through the core boundary (evenly driven by electrons and ions); "self-consistent" core boundary condition for the density (the plasma flux outgoing from the core boundary at the outer mid-plane is equal to the integral of the flux of neutrals that cross the core boundary); no sputtering from the tungsten solid surfaces, and a recycling coefficient of 1 for the ions. The transport coefficients were set as in ref. 7 , where they have been chosen for similarity with an H-mode ASDEX Upgrade discharge. ${ }^{[8]}$

A pump is present at the bottom of the machine, under the baffle, with an albedo set to 0.95 except for the third simulation ( 0.98 , to facilitate the onset of detachment); furthermore, a puff is also present in the private flux region, inserting $\mathrm{D}_{2}$ molecules at the temperature of $0.03 \mathrm{eV}$ with a rate of, respectively, $1 \times 10^{21}, 2 \times 10^{21}$, and $5 \times 10^{21} \mathrm{e}^{-} / \mathrm{s}$ for the first, second, and third simulation, respectively, named in the following as cases (a), (b), and (c).

Results from Soledge2D-Eirene in terms of plasma density, electron temperature, and ion temperature are shown in Figure 1.

As can be seen, owing to the low puff rate and to the absence of impurities to cool down the plasma, the electron (ion) temperature is quite high in case (a), with a peak value of $\sim 103 \mathrm{eV}(\sim 78 \mathrm{eV})$ on the outer strike point on the lower divertor. Increasing the puff rate makes it possible to reduce this peak value to $\sim 70 \mathrm{eV}(\sim 40 \mathrm{eV})$ for case (b), and finally below $\sim 3 \mathrm{eV}$ 
(a)
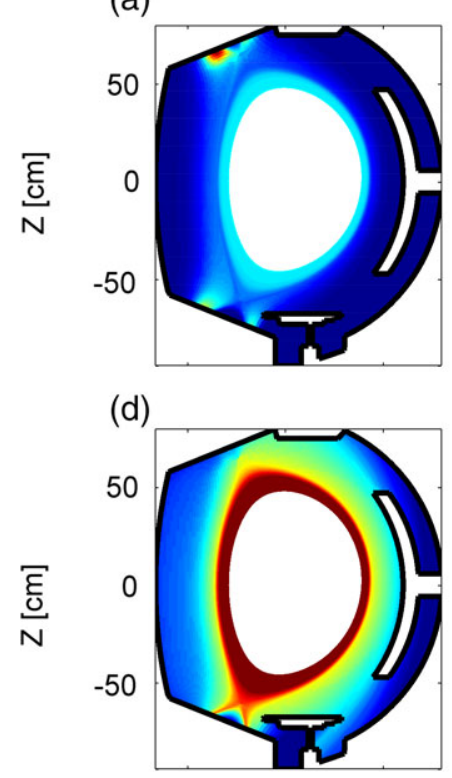

(g)

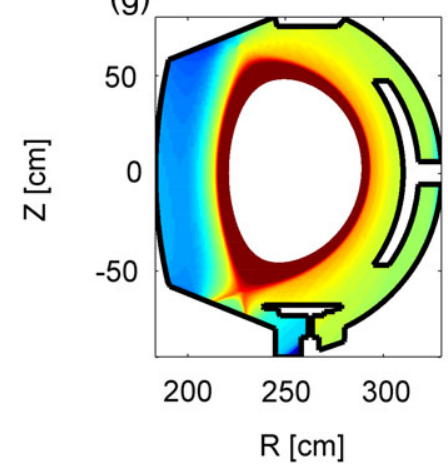

(b)
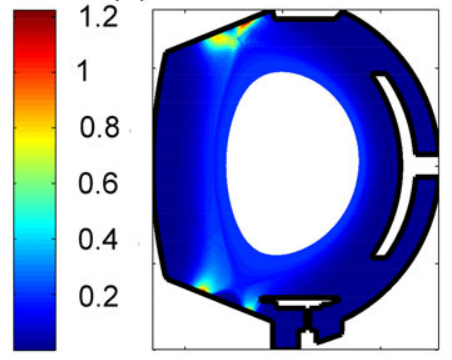

(e)

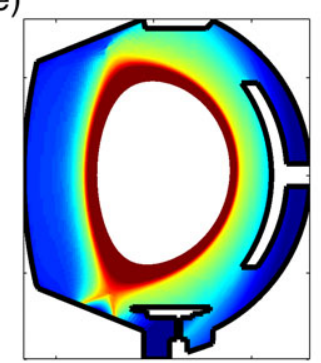

(h)

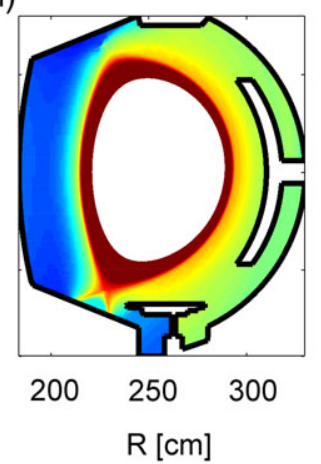

(c)
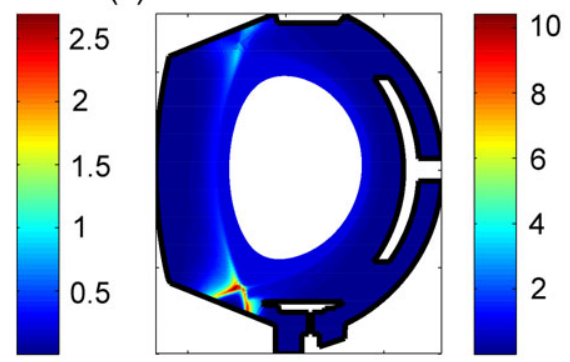

(f)

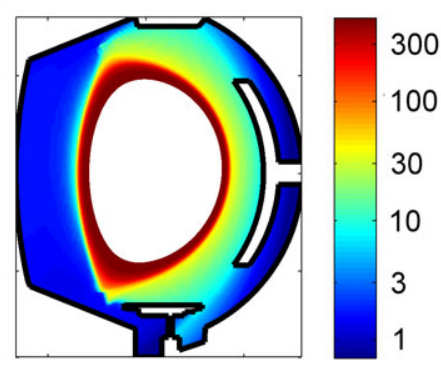

(i)

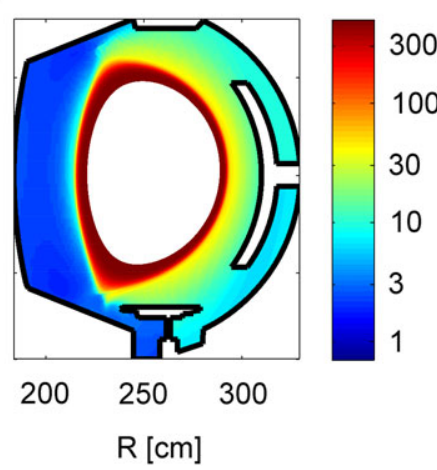

FIGURE 1 Plasma density $\left(10^{20} \mathrm{~m}^{-3}\right)(\mathrm{a}-\mathrm{c})$, electron temperature $(\mathrm{eV})(\mathrm{d}-\mathrm{f})$, and ion temperature $(\mathrm{eV})(\mathrm{g}-\mathrm{i})$ obtained by Soledge2D-Eirene

(in case c). At the same time, the plasma density increases with the puff rate, and the onset of volumetric recombination in case (c) progressively shifts the position of the density peak along the separatrix towards the x-point as the plasma detaches.

For case (a), the temperature seems to stay quite constant along the field line, indicative of a sheath-limited regime, with only a slight drop in front of the targets; in case (b), this drop in temperature at the targets gets larger as the divertor enters in a high recycling regime; finally, in case (c), a steep temperature gradient is found in the vicinity of the x-point, while it flattens in front of the targets. A similar behaviour is found in the upper divertor, even though it seems to be in a high recycling regime in case (a), and the onset of detachment is already visible in simulation (b).

Owing to the drop in temperature between cases (a) and (b), even though the peak particle flux increases by almost a factor of two (from $5.44 \times 10^{22}$ to $\left.1 \times 10^{23} \mathrm{~m}^{-2} \mathrm{~s}^{-1}\right)$, the peak heat flux on the outer target stays almost constant $\left(\sim 4 \mathrm{MW} / \mathrm{m}^{2}\right)$. In case (c), instead, the heat flux drops drastically to $0.7 \mathrm{MW} / \mathrm{m}^{2}$, as a consequence of volumetric recombination and collisions with neutral particles along the divertor legs.

In Figure 2, the density of atoms computed by Eirene is shown. In accordance with the Soledge2D results, in the first two cases the atom density increases at the targets as a result of the increase of the impinging ion flux, while in the upper divertor in case (b) and in both divertors in case (c) volumetric recombination floods the legs with neutral particles from the targets up to the $\mathrm{x}$-points.

\section{4 | FLUID NEUTRALS CODE SIMULATIONS}

Using the results obtained by Soledge2D-Eirene (i.e. plasma density, electron and ion temperature, and ion parallel velocity) as a fixed background, the fluid neutrals code was run for the three cases explored in the previous section. The simulations were run on the same triangular mesh and with the same recycling coefficients $R_{i}=R_{n}=1$ used by Eirene. 
(a)

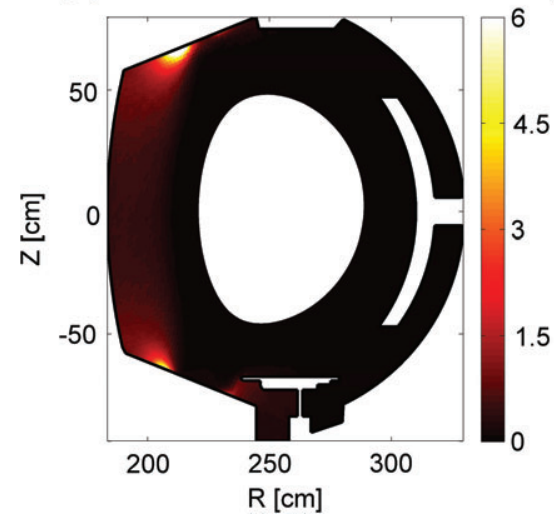

(b)

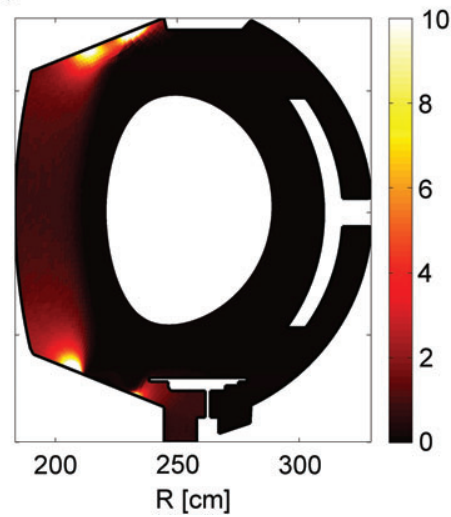

(c)

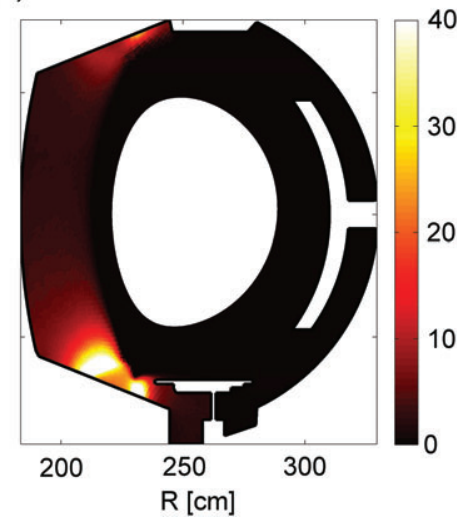

FIGURE 2 Atom density $\left(10^{18} \mathrm{~m}^{-3}\right)$ obtained by Eirene for the simulations with puff rates of 1,2 and $5 \times 10^{21} \mathrm{e}^{-/ \mathrm{s}}$ respectively

(a)

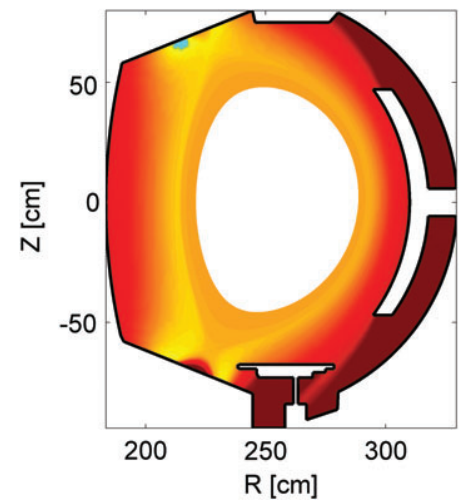

(b)

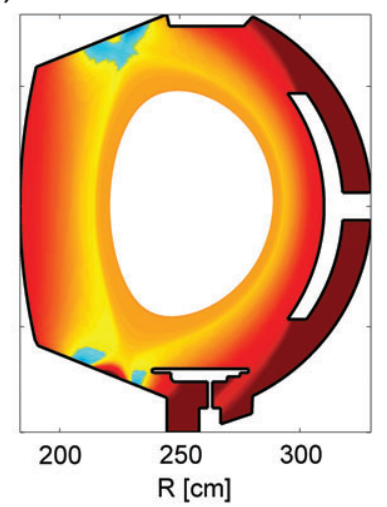

(c)

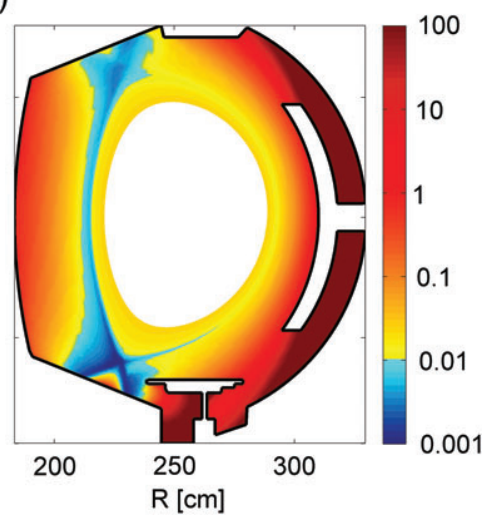

FIGURE 3 Knudsen number for D atoms for the three Soledge2D-Eirene simulations with puff rates of 1,2 and $5 \times 10^{21} \mathrm{e}^{-/ \mathrm{s}}$ respectively

It should be noted that a fluid description is usually taken as valid if the Knudsen number of a particle species, defined as $K n=\frac{\lambda}{L}=\frac{1}{\Sigma L}$ (where $\lambda$ is the mean free path of the neutral particles, $\Sigma$ is their total macroscopic cross-section, and $L$ is a macroscopic length scale), is $<0.01$. ${ }^{[9]}$

In Figure 3, we have plotted the Knudsen number for the atoms in the three cases. Here, the total macroscopic cross-section has been estimated as $\Sigma=\Sigma_{i z}+\Sigma_{r c}+\Sigma_{c x} \simeq \frac{n_{i}}{c_{s}}\left(\langle\sigma v\rangle_{i z}+\langle\sigma v\rangle_{r c}+\langle\sigma v\rangle_{c x}\right)$ and the macroscopic length scale has been taken as the tokamak minor radius $L=0.5 \mathrm{~m}$.

It is evident that the domain of validity of a fluid description, shown with blue shades in the figures, even though almost non-existent in case (a), gets larger with the puff rate as a result of the increasing density and decreasing temperatures.

As stated in Section 2, the fluid momentum equation is closed under the assumption of thermodynamic equilibrium between ions and atoms $\left(T_{n}=T_{i}\right)$. In Figure 4, the ratio of the atom temperature sampled by Eirene and the ion temperature is shown for the three simulations. If we forget the areas near the separatrix at the outer mid-plane and behind the antenna, where the atom temperature is quite noisy because of the fact that only a small number of particles actually scores in this locations, going from case (a) to case (c) the ratio gets closer to 1 as the charge exchange collisions tend to equalize the temperatures of ions and neutrals. This proves that the assumption, at least nearing detached conditions, can be justifiable.

Finally, because of the lack of puffs in the fluid code, the only source of atoms is recombination, either as recycling of the ion flux, plotted in Figure 5, or as volumetric reaction. However, since the fluid neutrals simulations are run in "standalone" and molecules are not taken into account, the puffs should not have a relevant impact: the total ion flux (and thus the recycled atom flux) on the wall is of the order of $\sim 2-4 \times 10^{23} \mathrm{D} / \mathrm{s}$, while the puff in the detached case is "only" $5 \times 10^{21} \mathrm{e}^{-} / \mathrm{s}$. Of course, this is not true anymore when fully coupled runs with Soledge2D are performed.

The results of the simulations are shown in Figure 6 in terms of neutral density, neutral particle source, and neutral parallel momentum source. For case (a), the particle source is always negative, indicating that the predominant process is ionization, due to the quite high plasma temperature, peaking at the two strike points; a similar behaviour is found for case (b), even though a positive source due to volumetric recombination is found on the upper divertor, especially at the high-field side; finally in case (c), the volumetric recombination takes over as the predominant process in the lower divertor as it detaches and the neutral density increases. For the parallel momentum source, cases (a) and (b) behave in a similar fashion, with neutral particles getting 

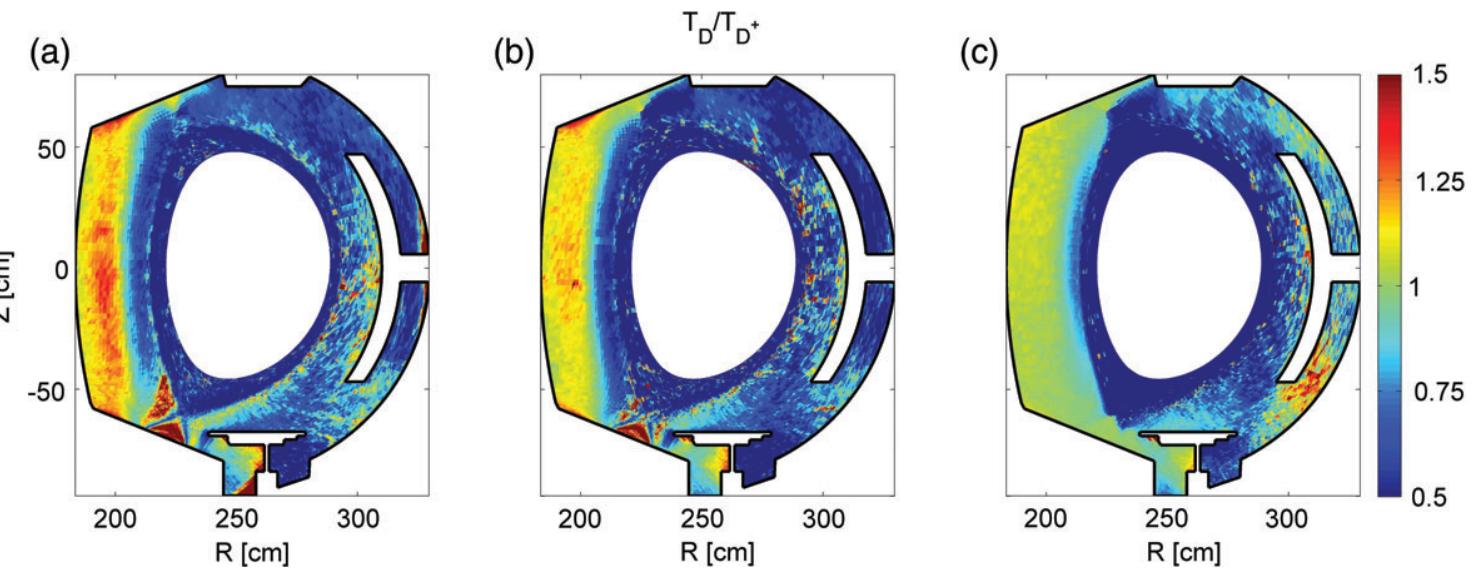

FIGURE 4 Ratio of the temperature of the atoms sampled by Eirene over the temperature of the ions computed by Soledge2D for the simulations with puff rates of 1,2 and $5 \times 10^{21} \mathrm{e}^{-/ \mathrm{s}}$ respectively

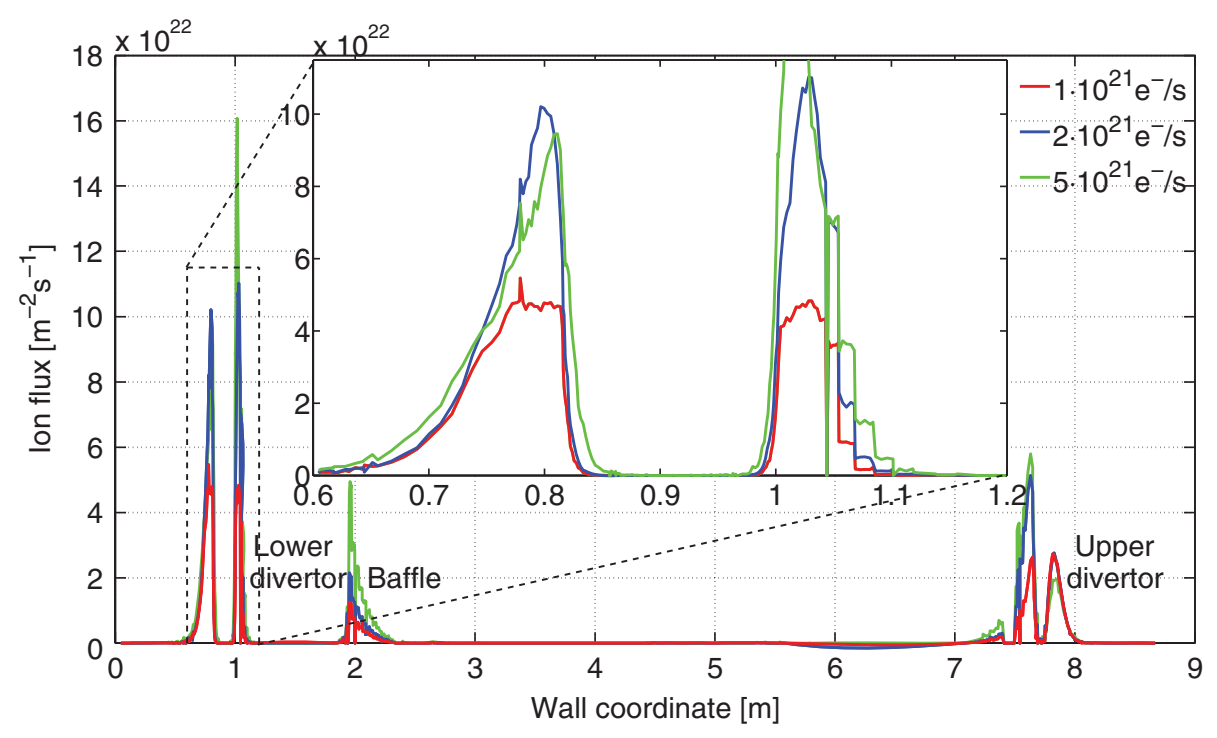

FIGURE 5 Ion flux impacting the solid walls

accelerated by the ion flux due to charge-exchange collisions in front of the targets; in case (c), again, it is instead the source due to volumetric recombination that predominates, accelerating the neutrals (and conversely, reducing the ion flux) all along the divertor legs from the x-point to the targets.

\section{5 | IMPACT OF MOLECULES}

When comparing with results obtained by Eirene, the neutral density computed by the fluid code seems to be about an order of magnitude lower, especially at the strike points, similar to what was found in ref. 10. A possible explanation may be the lack of molecules in the fluid code: as shown in Table 1, most of the molecular reactions not present result in the creation of D atoms; furthermore, as already explained in Section 2, only the fast fraction of the recycling flux is taken into account, thus effectively shifting the volumetric source of atoms due to Franck-Condon dissociation of the desorbing molecules to a surface source.

To test this hypothesis, another Soledge2D-Eirene simulation was run, with similar inputs but only $\mathrm{D}$ atoms this time. Furthermore, no puff was used in Eirene to make a comparison with the fluid code easier. The results reveal a very hot sheath-limited regime: the temperature at the outer target, in fact, reaches peak values of $\sim 245 \mathrm{eV}$ for the ions and $\sim 225 \mathrm{eV}$ for the electrons because of the relatively low density $\left(\sim 4 \times 10^{18} \mathrm{~m}^{-3}\right)$. The results obtained by Eirene in terms of atom density, atom particle source, and parallel momentum source are plotted in Figure 7.

Like before, the plasma fields have been used as background for the fluid code, and the comparison between Eirene and the fluid code is shown in Figure 8.

When comparing now with the Eirene results, it is evident from the red lines in Figure 9 that the discrepancy between the two codes along the separatrix decreases to a factor of $\sim 3$ at the peak value for the atom density; nonetheless, the code still underestimates the density at the target, and does not get the sharp gradient along the divertor leg. This, on the other hand, 

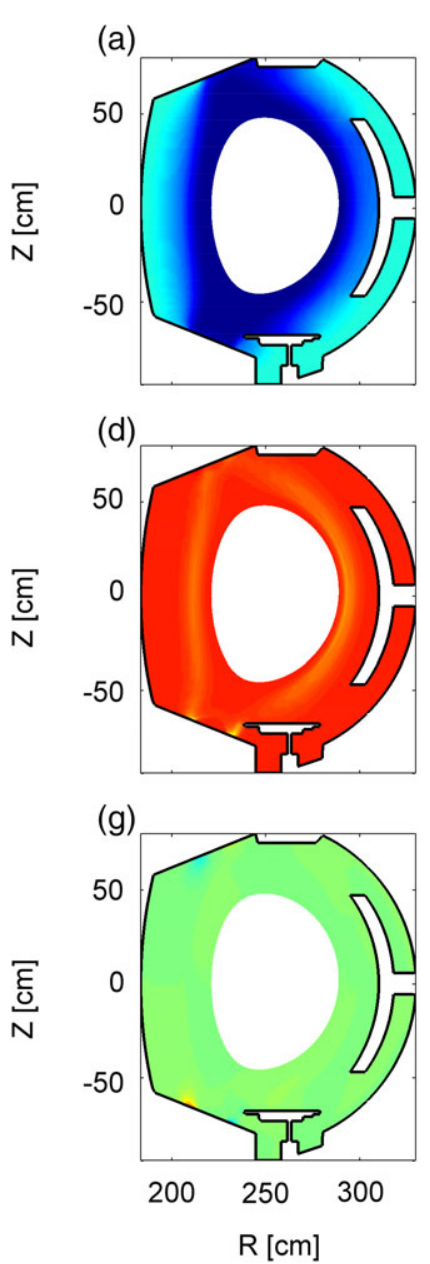

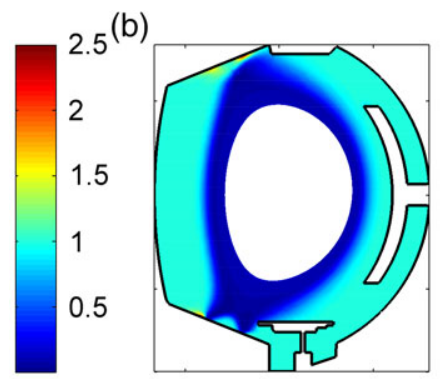

(e)
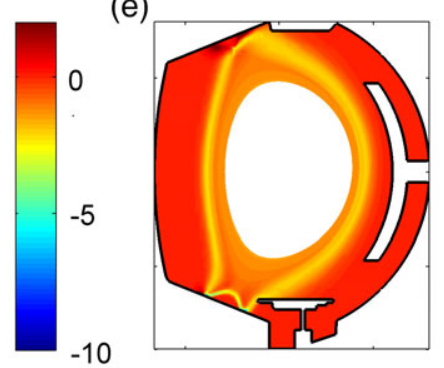

(h)
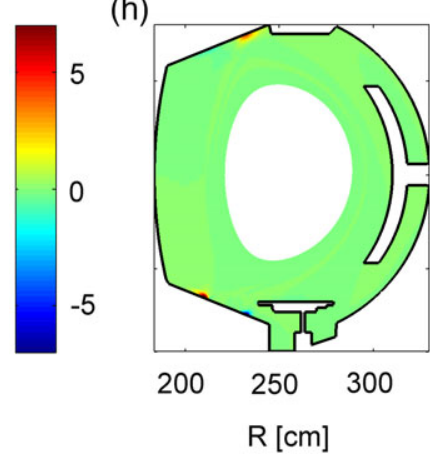
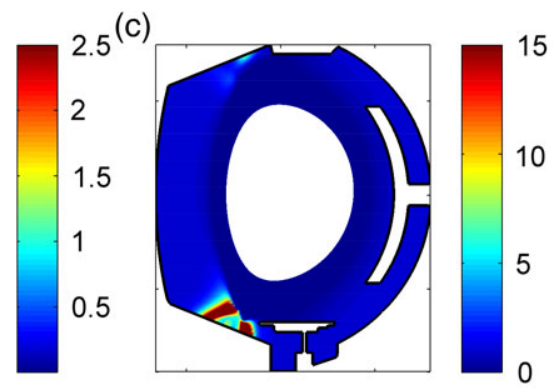

(f)
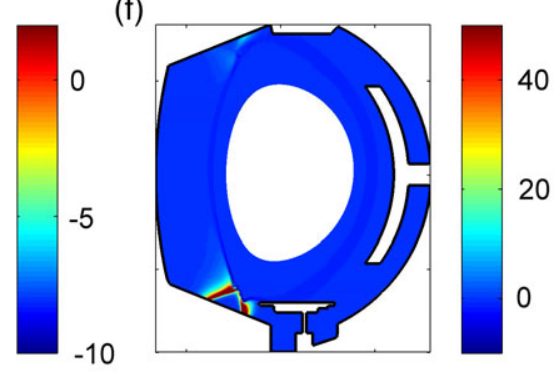

(i)

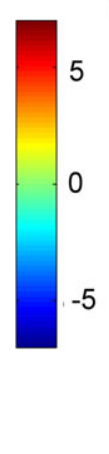

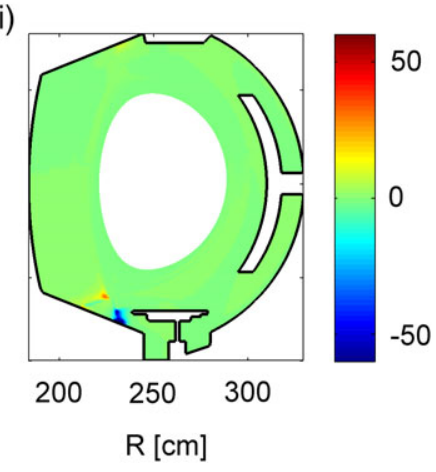

FIGURE 6 Neutral density $\left(10^{17} \mathrm{~m}^{-3}\right)(\mathrm{a}-\mathrm{c})$, neutral particle source $\left(10^{22} \mathrm{~m}^{-3} \mathrm{~s}^{-1}\right)(\mathrm{d}-\mathrm{f})$, and neutral parallel momentum source $\left(10^{27} \mathrm{~m}^{-2} \mathrm{~s}^{-2}\right)(\mathrm{g}-\mathrm{i})$ computed by the fluid code
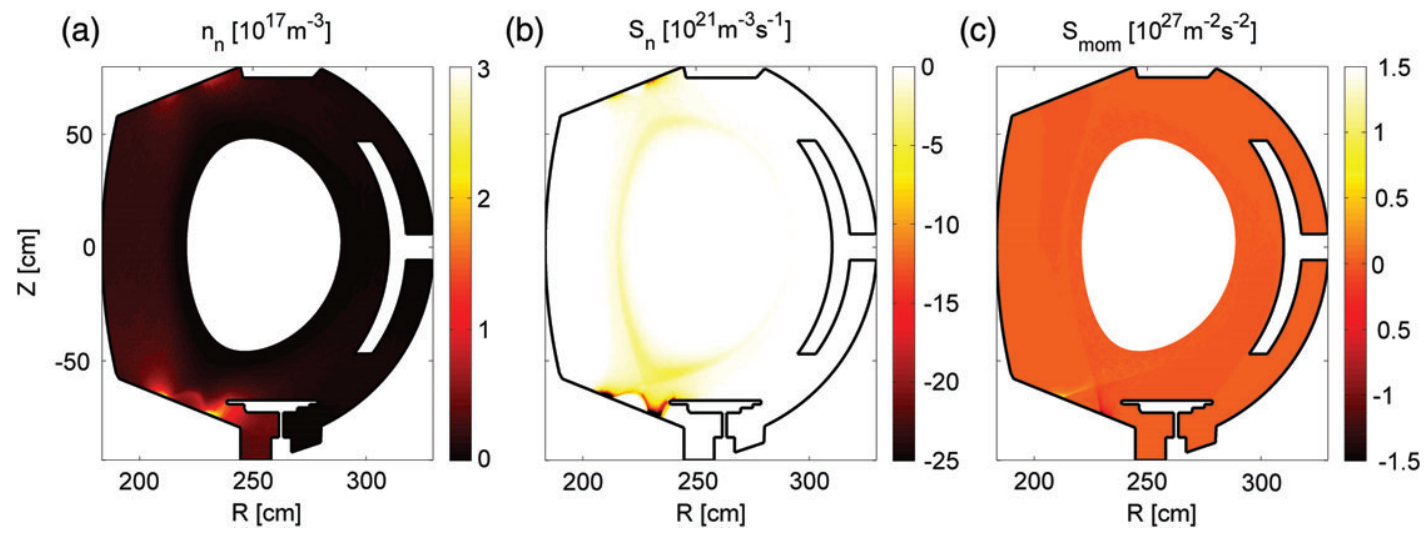

FIGURE 7 Atoms density $\left(10^{17} \mathrm{~m}^{-3}\right)(\mathrm{a})$, plasma particle source $\left(10^{21} \mathrm{~m}^{-3} \mathrm{~s}^{-1}\right)(\mathrm{b})$, and plasma parallel momentum source $\left(10^{27} \mathrm{~m}^{-2} \mathrm{~s}^{-2}\right)(\mathrm{c})$ obtained by Soledge2D-Eirene without molecules

can be explained by how the recycling boundary condition has been implemented in the fluid code. As already explained, the neutrals born of recycled ions have been assumed, for simplicity, to come out of the wall along the normal vector in the poloidal plane, with no toroidal component. On the other hand, Eirene expects a quite large fraction of the neutral velocity to be in the toroidal direction. This difference makes the fluid code overestimate the poloidal component of the velocity of the neutrals at the wall, as shown in Figure 9 with blue lines: because of this, the neutrals in the fluid code can penetrate further into the plasma before getting ionized, thus explaining the lower peak value at the wall and the slow gradient. The implementation of boundary conditions in accordance with the kinetic description of the neutrals has already been shown to be crucial to the accuracy of fluid models. ${ }^{[1]}$ 

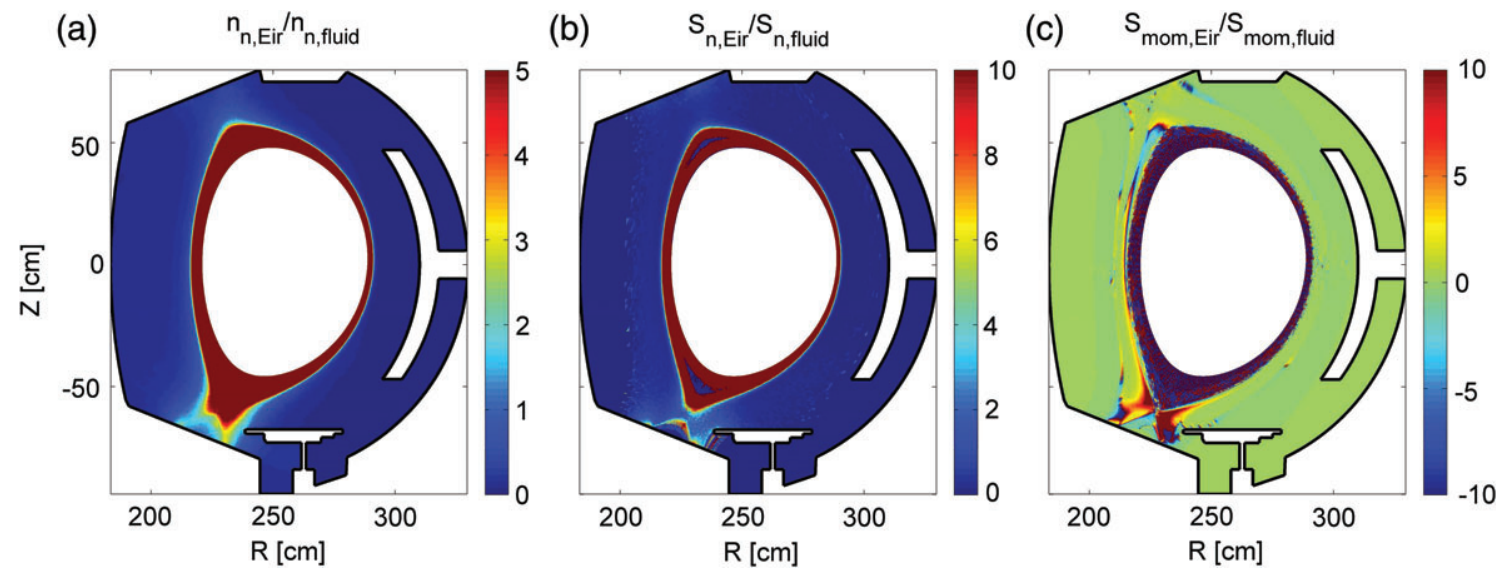

FIGURE 8 Ratio of atoms density (a), neutral particle source (b), and neutral parallel momentum source (c) obtained by Eirene with respect to the ones obtained by the fluid code

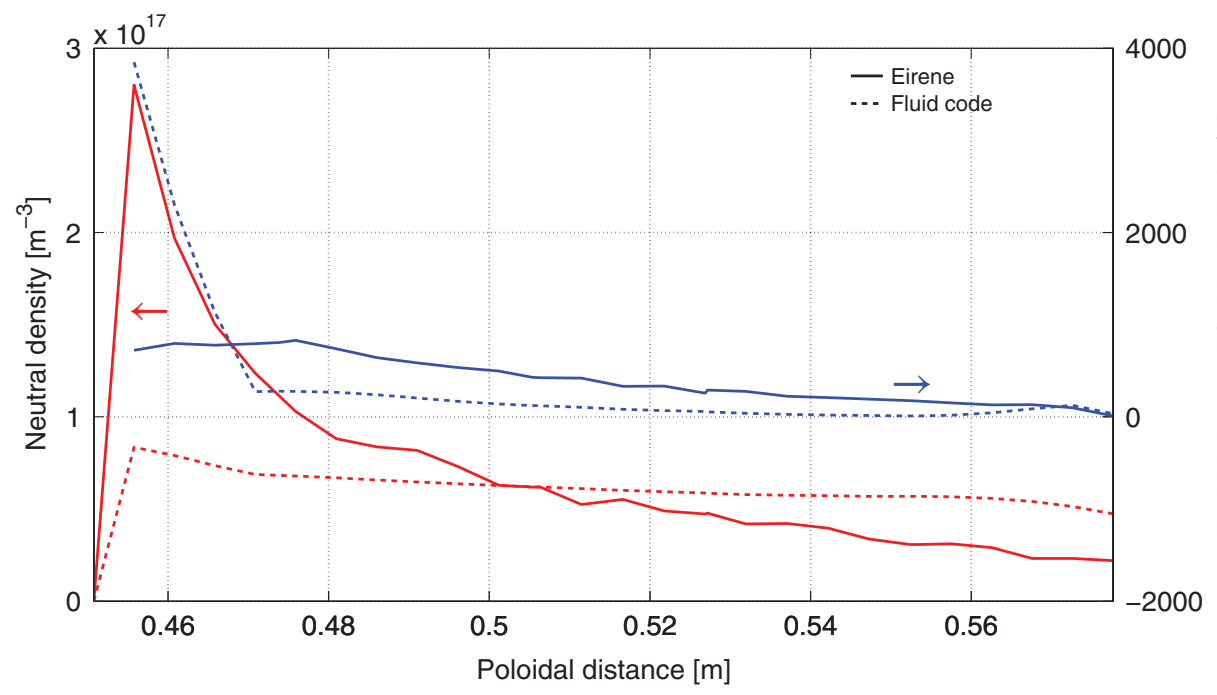

FIGURE 9 Atoms density $\left(\mathrm{m}^{-3}\right)$ (in red) and poloidal component of neutral fluid velocity $(\mathrm{m} / \mathrm{s})$ (in blue) along the separatrix, from the wall (at $0.45 \mathrm{~m}$, on the left) to the x-point (at $0.58 \mathrm{~m}$, on the right). Solid lines are for the results obtained by Eirene, and dashed lines are the results obtained by the fluid code

Last but not least, the Knudsen number for the atoms in the case just explored is quite high owing to the high temperature, and thus a fluid description should not be taken as acceptable.

\section{6 | CONCLUSIONS}

A new fluid code has been developed for Soledge2D-Eirene. This code solves Euler-like equations for hydrogen atoms, taking into account a reduced set of reactions with the background plasma. A further neutral source is due to recycling of the ion fluxes on the solid walls, even though the implementation assumes the simplification of zero toroidal component for the neutrals coming out of the wall and no "slow" recycling through molecules. The code is able to reproduce the distribution of the neutrals even in complex and realistic geometries, and over a wide range of plasma background conditions, from sheath-limited to detached regimes. Nonetheless, some discrepancies are found when comparing the results of the fluid code with respect to the results obtained by Eirene, but these differences can be explained as due to the simplification introduced in the model. Finally, it should be remembered that a fluid description can be employed only in low Knudsen number $(<0.01)$ areas, but in most of the domain the neutrals behave kinetically, thus showing the need of a hybrid (kinetic-fluid) description to model the neutrals.

\section{ACKNOWLEDGEMENTS}

This work was carried out within the framework of the Eurofusion Consortium, and has received funding from the Euratom research and training program 2014-2018 under grant agreement No. 633053. The views and opinions expressed herein do not necessarily reflect those of the European Commission. This work was granted access to the HPC resources of Aix-Marseille Université financed by the project Equip@ Meso (ANR-10-EQPX-29-01) of the program "Investissements d'Avenir" supervised by the Agence Nationale pour la Recherche. 
ORCID

M. Valentinuzzi (1) http://orcid.org/0000-0001-6364-6640

\section{REFERENCES}

[1] H. Bufferand, G. Ciraolo, Y. Marandet, J. Bucalossi, P. Ghendrih, J. Gunn, N. Mellet, P. Tamain, R. Leybros, N. Fedorczak, F. Schwander, E. Serre, Nucl. Fusion 2015, 55, 053025 .

[2] D. Reiter, M. Baelmans, P. Börner, Fusion Sci. Technol. 2005, 47, 172.

[3] V. Kotov, D. Reiter, R. A. Pitts, S. Jachmich, A. Huber, D. P. Coster and JET-EFDA contributors, Plasma Phys. Control. Fusion 2008, 50, 105012.

[4] G. Giorgiani, S. Fernández-Méndez, A. Huerta, Comput. Fluids 2014, 98, 196.

[5] B. Cockburn, G. Kanschat, D. Schötzau, Comput. Fluids 2005, 34, 491.

[6] C. Bourdelle et al., Nucl. Fusion 2015, 55, 063017.

[7] G. Ciraolo, H. Bufferand, J. Bucalossi, P. Ghendrih, P. Tamain, Y. Marandet, M. Valentinuzzi, J. Denis, N. Fedorczak, E. Hodille, N. Mellet, B. Pegourie, C. Grisolia, C. Bourdelle, E. Tsitrone, D. Galassi, R. Leybros, G. Giorgiani, E. Serre, Nucl. Mater. Energy 2017, $12,187$.

[8] A. V. Chankin, D. P. Coster, R. Dux, C. Fuchs, G. Haas, A. Herrmann, L. D. Horton, A. Kallenbach, M. Kaufmann, C. Konz, K. Lackner, C. Maggi, H. W. Müller, J. Neuhauser, R. Pugno, M. Reich, W. Schneider, Plasma Phys. Control. Fusion 2006, 48, 839.

[9] T. E. Schwartzentruber, L. C. Scalabrin, I. D. Boyd, J. Spacecraft Rockets 2008, 45, 1196.

[10] K. Hoshino, M. Toma, A. Hatayama, D. P. Coster, X. Bonnin, R. Schneider, H. Kawashima, N. Asakura, Y. Suzuki, Contrib. Plasma Phys. 2008, 48(1-3), 136.

[11] N. Horsten, W. Dekeyser, G. Samaey, M. Baelmas, Nucl. Mater. Energy 2017, 12, 869. 